\title{
Hepatocyte nuclear factor 1 binding element within the promoter of microsomal triglyceride transfer protein (MTTP) gene is crucial for MTTP basal expression and insulin responsiveness
}

\author{
Wo-Shing Au, Liwei Lu', Chung-Man Yeung, Ching-Chiu Liu, Oscar G Wong, Lihui Lai ${ }^{2}$, \\ Hsiang-fu Kung ${ }^{3}$ and Marie C Lin
}

Department of Chemistry, Institute of Molecular Technology for Drug Discovery and Synthesis, The University of Hong Kong, Room 8N-11, Kadoorie Biological Science Building, Pokfulam Road, Hong Kong, China

${ }^{1}$ Department of Pathology, The University of Hong Kong, Hong Kong, China

${ }^{2}$ Institute of Molecular and Chemical Biology, East China Normal University, Shanghai 200062, China

${ }^{3}$ Centre of Emerging Infectious Diseases, The Chinese University of Hong Kong, Hong Kong, China

(Correspondence should be addressed to M C Lin; Email: mcllin@hkusua.hku.hk)

\begin{abstract}
Insulin inhibits the transcription of the microsomal triglyceride transfer protein (MTTP), which plays a pivotal role in lipoprotein assembly and secretion. Here, we provide evidence that a hepatocyte nuclear factor 1 binding element (HNF1A element) within the MTTP promoter serves as a novel negative insulin-responsive element. Deletion/mutation mapping of the MTTP gene promoter identified a modified HNF1A element that is crucial to the negative insulin effect. Chimeric promoter containing this HNF1A element and minimal TEAD1 promoter also responded negatively toward insulin treatment. Gel shift assay demonstrated that HNF1A but not HNF1B binds to this element. Enforced expression of HNF1A was sufficient to reconstitute the negative insulin responsiveness of MTTP promoter in TM4SF1 myocytes that are HNF1A negative. Furthermore, replacing this element with consensus HNF1A element preserved the negative insulin response, suggesting that negative insulin responsiveness is a generic characteristic of HNF1A element. Given that many genes implicated in diabetes contain HNF1A element, the potential regulation of these genes by insulin via HNF1A element may provide important clues for the manifestation and treatment of diabetic metabolic syndromes.
\end{abstract}

Journal of Molecular Endocrinology (2008) 41, 229-238

\section{Introduction}

Coupling with insulin receptor, insulin recruits a web of signaling molecules and/or transcription factors to relay the transmission, amplification, and integration of intracellular signals (Saltiel \& Kahn 2001). Propagation of these intracellular signals relies upon the timely interactions of signaling messengers, which are of protein, lipid, and DNAs in origin. Knowledge of the functional interactions among these molecules is central to unraveling the molecular wisdom of insulin action. Although various insulin signal transduction pathways have been extensively studied, the nuclear actions of insulin, in particular transcriptional regulation, are not well understood.

Insulin regulates the expression of more than 150 genes in both positive and negative manner (O'Brien et al. 2001, Mounier \& Posner 2006). Although the majority of these genes are positively regulated by insulin, 18 genes are found to be negatively regulated (Mounier \& Posner 2006). One of the genes whose transcription is negatively regulated by insulin is the microsomal triglyceride transfer protein $(M T T P)$ gene. MTTP regulates the production of apolipoprotein $\beta$-containing lipoproteins in both the liver and intestine (Wetterau et al. 1997, Hussain et al. 2003, Shoulders \& Shelness 2005). Human genetic linkage analyses (Austin et al. 1998, Rainwater et al. 1999) and functional studies of exon and promoter polymorphisms of MTTP (Ledmyr et al. 2002, 2004, 2006, Rubin et al. 2008) have suggested an association between the MTTP gene and diabetic dyslipidemia.

Previous studies from our laboratory and many others have demonstrated that MTTP is regulated mainly at transcription level (Hagan et al. 1994, Sharp et al. 1994, Lin et al. 1997, Sato et al. 1999) and that high-fat diets as well as diabetic conditions are associated with elevated hepatic and intestinal MTTP expressions (Lin et al. 1994, Kuriyama et al. 1998, Taghibiglou et al. 2000). Insulin serves as an important negative regulator of MTTP gene transcription (Lin et al. 1995, Au et al. 2003). However, the negative insulin-responsive element (NIRE) remains to be identified. 
In this study, we provided the first evidence that a functional hepatocyte nuclear factor 1 binding element (HNF1A element) in the MTTP promoter $(-110 /-98 \mathrm{bp})$ is a novel NIRE. Although the sequence of MTTP HNF1A element differs from that of the consensus HNF1A element sequence, we showed that they are functionally equivalent in terms of insulin response, suggesting that the negative insulin responsiveness is a generic characteristic of HNF1A element. Since many diabetes-affected genes contain HNF1A element, their potential regulation by insulin might yield important clues about the manifestation and the treatment of diabetic metabolic syndromes.

\section{Materials and methods}

\section{Plasmids construction}

Various lengths of human MTTP promoter (MTTP-612, $-250,-142,-115,-102,-76 /+86 \mathrm{bp}$, relative to the transcription start site) were amplified from human genomic library (Clontech). The mutant M0TTP promoters (MTTP-142m1, -142m2, -142m3, -142m4) were generated by PCR-based mutagenesis with mutations $\mathrm{A} \rightarrow \mathrm{C}$ and $\mathrm{T} \rightarrow \mathrm{G}$. The mutant MTTP promoters containing the mutated (MTTP-250mHNF1A; Bonham et al. 2000) and consensus (MTTP250-cHNF1A; Ryffel 2001) HNF1A sites were generated by overlapping PCR as described in the reference. These fragments were tailored to contain $K p n I$ and $B g I I I$ sites at the $5^{\prime}$ and $3^{\prime}$ ends respectively, and cloned into the respective sites of SDHC vector (SDHC-Basic; Promega). The fusion promoters constructs were generated by cloning the double-stranded (DS) oligonucleotides representing the MTTP promoter sequence $(-127 /-72 \mathrm{bp}), 1 \times / 4 \times$ the MTTP HNF1A element $(-111 /-94 \mathrm{bp})$, the consensus HNF1A element (Ryffel 2001), or the mutated HNF1A element (as suggested by (Bonham et al. 2000)) into the SDHC-TEAD1 backbone (SDHC-promoter, Promega) at $K p n \mathrm{I} / \mathrm{XhoI}$ sites. The expression vector encoding fulllength human HNF1A or HNF1B was a generous gift from Dr Takamatsu Nobuhiko (Ono et al. 2001) and Dr Crabtree respectively. All clones were verified by automated DNA sequencing.

\section{Cell culture}

HepG2 hepatoma cells and TM4SF1 muscle cells (ATCC) were cultured at $37^{\circ} \mathrm{C}$ in minimum essential medium (MEM) (containing $2 \mathrm{mM}$ nonessential amino acids, $2 \mathrm{mM}$ sodium pyruvate, $1.5 \mathrm{~g} / 1$ sodium bicarbonate, $100 \mathrm{U} / \mathrm{ml}$ penicillin, $100 \mu \mathrm{g} / \mathrm{ml}$ streptomycin, $10 \% \mathrm{FBS}$, and supplemented with $0.3 \mathrm{mg} / \mathrm{ml} \mathrm{L-glutamine)}$ and DMEM (containing 4.5 g/l glucose, $100 \mathrm{U} / \mathrm{ml}$ penicillin,
$100 \mu \mathrm{g} / \mathrm{ml}$ streptomycin, and $10 \% \mathrm{FBS}$ ) respectively in a humidified $5 \% \mathrm{CO}_{2}$ incubator. The cells received fresh medium every 2-3 days.

\section{Transfection and reporter assay}

Cells were seeded at $\sim 70 \%$ density in 12-well plates. A DNA/liposome mix containing $1.5 \mu \mathrm{g}$ LipofectAMINE 2000 (Invitrogen), 0.2-0.5 $\mu$ g wild-type or mutant MTTP promoter-reporter plasmid, $0.01 \mu \mathrm{g}$ PRLTEAD1, and $0 \cdot 1-0.5 \mu \mathrm{g}$ expression plasmid (if necessary) DNA per well were used. The PRL-TEAD1 plasmid (Promega) coding for Renilla luciferase was co-transfected to serve as an internal control to normalize the transfection efficiency. Transfections were allowed to proceed for $6 \mathrm{~h}$ in the absence of serum, and the cells were then recovered in medium supplemented with reduced serum level $(2.5 \%)$ for $16 \mathrm{~h}$, followed by $100 \mathrm{nM}$ insulin (bovine, from CalBiochem, Merck KGnA, Darmstadt, Germany) challenge for $24 \mathrm{~h}$. Afterward, cells were lysed with passive lysis buffer (Promega) and luciferase activities were assayed using a dual luciferase reporter system (Promega), and were quantified in a Lumat LB 9507 luminometer (Berthold, Technologies, Bad Wilbad, Germany).

\section{Western blot analysis}

Cells were harvested and lysed in lysis buffer $(20 \mathrm{mM}$ Tris$\mathrm{HCl}(\mathrm{pH} 7 \cdot 4), 50 \mathrm{mM} \mathrm{NaCl}, 1 \mathrm{mM}$ EDTA, 1 mM EGTA, $1 \%$ Tritron X-100, $25 \mathrm{mM} \mathrm{NaF}, 1 \mathrm{mM} \mathrm{Na} 3 \mathrm{VO}_{4}, 1 \mathrm{mM}$ phenylmethylsulfonyl fluoride (PMSF), $5 \mu \mathrm{g} / \mathrm{ml}$ leupeptin, and $5 \mu \mathrm{g} / \mathrm{ml}$ aprotinin). Protein samples $(20 \mu \mathrm{g}$ each) were resolved by $8 \%$ SDS-PAGE and transferred to polyvinylidene difluoride membrane $(0.45 \mu \mathrm{m}$ pore size, Immobilon-P, Millipore, Billerica, MA, USA). The blot was blocked with $5 \%$ skim milk, followed by incubation with specific antibodies against HNF1A or HNF1B, and $\alpha$-tubulin, as well as secondary antibodies conjugated with horse-radish peroxidase (HRP). Immunoreactive bands were detected using the ECL ${ }^{\mathrm{TM}}$ Western blotting detection reagents (Amersham Biosciences, Piscataway, NJ, USA). A representative blot from at least two separate experiments was shown.

\section{Electrophoretic mobility shift assay (EMSA)}

Nuclear extract was prepared from HepG2 cells according to the principle described by Dignam (25). Freshly prepared cell pellet $\left(>1 \times 10^{7}\right.$ cells) resuspended in $5 \times$ packed cell volume (PCV) of cold hypotonic buffer (10 mM HEPES ( $\mathrm{pH} 7.9), 1.5 \mathrm{mM}$ $\mathrm{MgCl}_{2}, 10 \mathrm{mM} \mathrm{KCl}, 0 \cdot 1 \mathrm{mM}$ dithiothreitol (DTT), and $0.5 \mathrm{mM}$ PMSF) was kept on ice for $15 \mathrm{~min}$. Nonidet P-40 was added to the cells at a final concentration of $0 \cdot 2 \%$. 
The suspension was pelleted at $10000 \mathrm{~g}$ at $4{ }^{\circ} \mathrm{C}$ and was resuspended in $2 / 3 \mathrm{PCV}$ of cold extraction buffer (HEPES (pH 7.9), 25\% (v/v) glycerol, $420 \mathrm{mM} \mathrm{NaCl,}$ $1.5 \mathrm{mM} \mathrm{MgCl}_{2}, 0.2 \mathrm{mM}$ EDTA, $0.1 \mathrm{mM}$ DTT, and $0.5 \mathrm{mM}$ PMSF). After $30 \mathrm{~min}$ incubation with agitation, cells were pelleted. Supernatant containing the DNAbinding proteins was divided into aliquots and stored at $-70^{\circ} \mathrm{C}$. DS oligonucleotide probe and competitors were prepared by annealing the complementary oligonucleotides. The probe $(31 \mathrm{fmol})$, end labeled with digoxigenin (DIG)-conjugated ddUTP using terminal transferase (Roche), was incubated with HepG2 nuclear extract $(5 \mu \mathrm{g})$ in a final reaction volume of $20 \mu \mathrm{l}$ containing $20 \mathrm{mM}$ HEPES ( $\mathrm{pH} 7 \cdot 6$ ), $1 \mathrm{mM}$ EDTA, $10 \mathrm{mM}\left(\mathrm{NH}_{4}\right)_{2} \mathrm{SO}_{4}, 1 \mathrm{mM}$ DTT, $0 \cdot 2 \%$ Tween 20 (v/v), $30 \mathrm{mM} \mathrm{KCl}, 0.5 \mu \mathrm{g}$ poly[d(I-C) ], and $0.05 \mu \mathrm{g}$ poly L-lysine. For competition and supershift assay, 100-fold molar excess of unlabeled DS oligonucleotides and antibodies were included in the reaction mixture. The mixture was incubated on ice for $30 \mathrm{~min}$ and then resolved by $5 \%$ native polyacrylamide gel. After electrophoresis, the oligonucleotides were transferred to Hybond $\mathrm{N}+$ membrane (Amersham Bioscience) using electroblotting method, and fixed by u.v. crosslinking. Blocking and probing using anti-DIG antibody with alkaline phosphatase (AP) conjugate and washes were conducted. Mobility shift signals were detected using chloro-5-substituted adamantyl-1,2-dioxetane phosphate (CSPD) reagent (Roche). A representative blot from at least two separate experiments was shown.

\section{Co-immunoprecipitation assay}

Nuclear extract was prepared as described in the previous section. Fifty microgram of the nuclear extract was diluted in $100 \mu \mathrm{l}$ PBS supplemented with protease inhibitor mix and was incubated under agitation with $5 \mu \mathrm{l}$ of goat antiHNF1A antibody (200 $\mu \mathrm{g} / \mathrm{ml}$; Santa Cruz Biotechnology, Santa Cruz, CA, USA) at $4{ }^{\circ} \mathrm{C}$ for $1 \mathrm{~h}$. About $10 \mu \mathrm{l}$ of recProtein G sepharose 4B (Zymed Laboratories, San Francisco, CA, USA) was then added to the mixture and the mixture was agitated at $4{ }^{\circ} \mathrm{C}$ for $1 \mathrm{~h}$. The precipitating protein complex was pelleted by centrifugation at $10000 \mathrm{~g}$ for $30 \mathrm{~s}$ at $4{ }^{\circ} \mathrm{C}$. The pellet was rinsed with PBS supplemented with protease inhibitor mix for five times and was resuspended in $20 \mu \mathrm{l}$ of $1 \times$ protein sample buffer. The suspension was boiled for $5 \mathrm{~min}$ and was resolved by SDS-PAGE after removal of debris by brief centrifugation as described in the previous section.

\section{Statistical analysis}

Statistical analysis was performed using two-tailed Student's $t$-test, and $P<0 \cdot 05$ was considered significant.
Data are expressed as the mean \pm s.E.M. of results obtained from three independent experiments.

\section{Results}

\section{HNF1A element is required for both basal activation and the negative insulin responsiveness of the MTTP promoter activity}

We have previously shown that insulin decreases hepatic MTTP mRNA levels (Lin et al. 1995) and that transcriptional regulation is the main force for this insulin effect (Au et al. 2003). To map the NIRE, we tested the insulin effect on reporter constructs containing a series of the MTTP promoter with sequential $5^{\prime}$ deletions in HepG2 cells. Figure 1A shows a schematic of the structure of the human MTTP promoter. As shown in Fig. 1B, $50 \%$ reduction in promoter activity after insulin treatment was seen in the constructs containing $-612,-250,-142$, and -115 to $+86 \mathrm{bp}$ (relative to transcription start site) of the MTTP promoter (MTTP-612, -250, -142, and -115), and this magnitude of inhibition is consistent with the reported insulin effect (Lin et al. 1995). However, further deletions to -102 or -76 bp (MTTP-102, -76) abrogated the insulin inhibition as well as the basal promoter activity, suggesting the presence of an NIRE and a basal activation element located at the downstream of $-115 \mathrm{bp}$ region.

To locate the NIRE and basal activation element, we generated a panel of mutation constructs, which covers the three predicted cis-elements resided in the -124 to $-76 \mathrm{bp}$ region: the sterol-responsive element (SRE, -124 to $-112 \mathrm{bp}$; MTTP-142m1), HNF1A element ( -110 to $-98 \mathrm{bp}$; MTTP-142m2, -142m3), and HNF4A element ( -95 to $-83 \mathrm{bp}$; MTTP-142m4). As shown in Fig. 1C, MTTP-142m1 and MTTP-142m4 mutants maintained the insulin effect, which is consistent with a previous report that SRE is not responsible for the insulin effect on MTTP promoter activity (Sato et al. 1999). Most importantly, insulin-mediated inhibition was abolished in MTTP-142m2 or MTTP-142m3 mutants, and with a simultaneous reduction in the basal promoter activity. These data, in accordance with the deletion analysis, indicated that the HNF1A element, which encompasses -110 to $-98 \mathrm{bp}$ of the MTTP promoter, is essential for both negative insulin effect and basal promoter activity.

\section{The HNF1A element is able to confer insulin respon- siveness and promoter activation in heterologous promoter}

To determine whether insulin signals through HNF1A element or just requires the element to elevate basal 
A

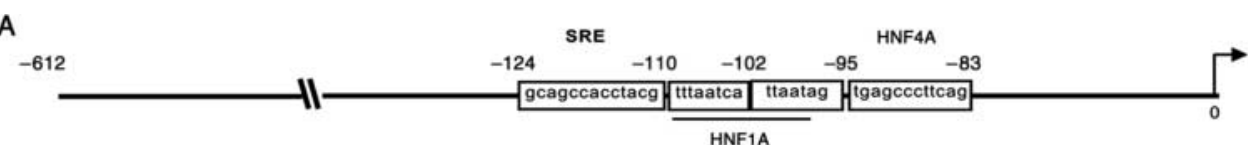

B
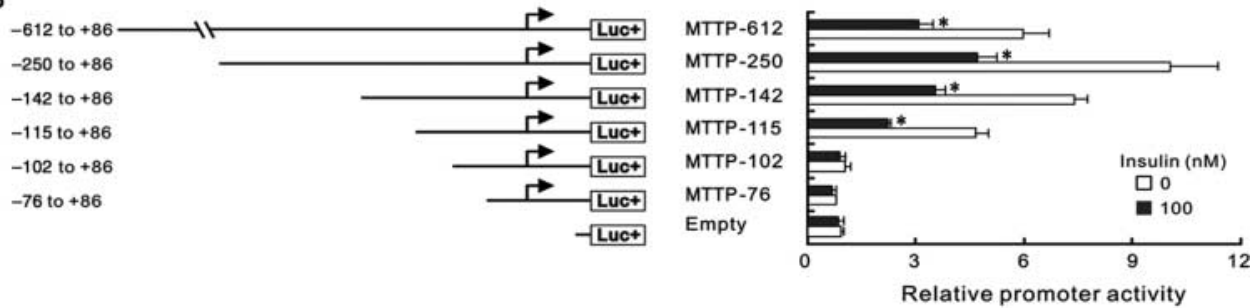

C
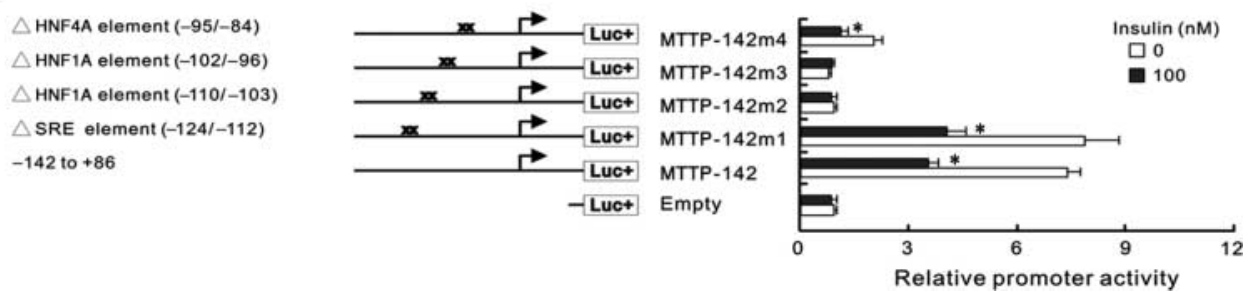

Figure $1 \mathrm{HNF} 1 \mathrm{~A}$ element mediates basal activation and negative insulin response of the MTTP promoter. (A) Schematic of the structure of the human MTTP promoter. (B) Deletion mapping and (C) mutation mapping of NIRE. HepG2 cells were transiently transfected with the indicated deletion/mutation luciferase (luc +) reporter constructs $(0.5 \mu \mathrm{g})$, recovered in reduced serum $(2.5 \%)$ medium for $16 \mathrm{~h}$, and then treated with or without $100 \mathrm{nM}$ insulin in serum-free medium for $24 \mathrm{~h}$. The cells were then harvested and assayed for promoter activities. Relative promoter activity was calculated as the ratio of normalized RPUSD1 of different treated or untreated promoters to that of the untreated vector control or empty vector. $\mathrm{xx}$ denotes mutation $(A \rightarrow C$ and $G \rightarrow T$ of indicated element). *Denotes significant difference from untreated counterpart $(P<0 \cdot 05)$.

promoter activity such that the negative effect of insulin is more apparent, we generated several chimeric promoters by fusing the MTTP promoter containing SRE, HNF1A, and HNF4A binding sequences $(-127$ to $-72 \mathrm{bp}$ ) with TEAD1 minimal promoter and tested their insulin responsiveness in HepG2 cells. As shown in
Fig. 2, the presence of the MTTP promoter sequence ( -127 to $-72 \mathrm{bp}$, MTTP-reg), one $(1 \times \mathrm{HNF} 1 \mathrm{~A})$ or four tandem copies $(4 \times$ HNF1A) of HNF1A element significantly enhanced the promoter activity over the TEAD1 vector control, supporting its role as a basal activating element. In addition, insulin significantly suppressed
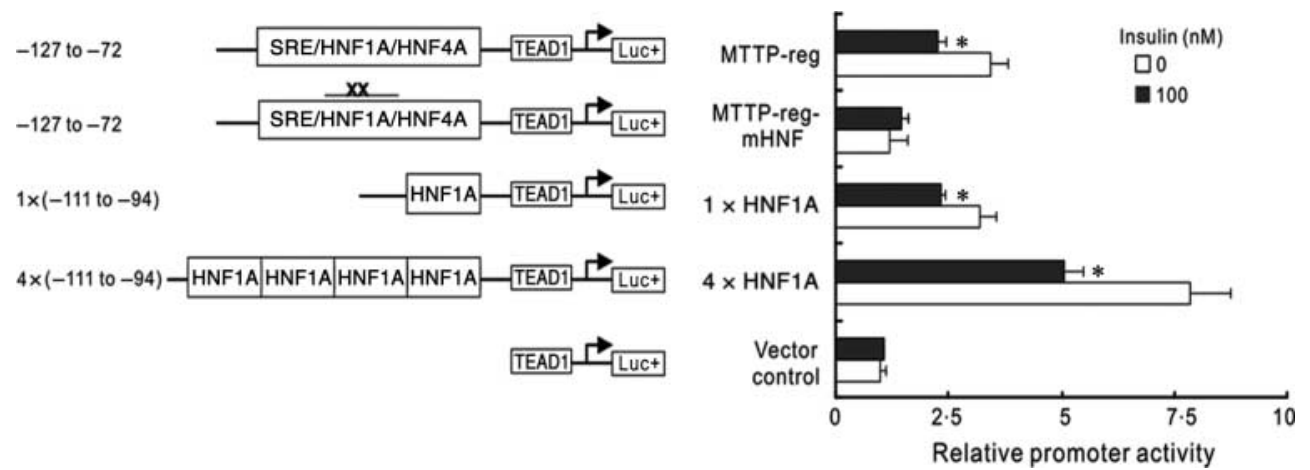

Figure 2 Fusion of HNF1A element with heterologous promoter confers activation and negative insulin response. HepG2 cells were transiently transfected with the indicated fusion promoter (luc + ) reporter construct $(0.3 \mu \mathrm{g})$, recovered in reduced serum $(2.5 \%)$ medium for $16 \mathrm{~h}$, and treated with or without $100 \mathrm{nM}$ insulin in serum-free medium for $24 \mathrm{~h}$. The cells were then harvested and assayed for promoter activities. Relative promoter activity was calculated as the ratio of normalized RPUSD1 of different treated or untreated promoters to that of the untreated vector control or empty vector. $x x$ indicates inactivating mutation. HNF1A, HNF1A element; HNF4A, HNF4A element; SRE, SRE element. *Denotes significant difference from untreated counterpart $(P<0.05)$. 
the promoter activity of MTTP-reg, $1 \times$ HNF1A, and $4 \times$ HNF1A by 35,30 , and $40 \%$ respectively. Mutation of the HNF1A motif (MTTP-reg-mHNF1A) abolished both the basal activation activity and insulin responsiveness. These results suggest that HNF1A element alone is sufficient to confer both the promoter basal activity and the negative insulin responsiveness.

\section{HNF1A binds to the HNF1A element in the MTTP promoter}

We conducted EMSA to identify the transcription factor(s) that bind(s) to this NIRE. Sequences of the oligonucleotides are shown in Fig. 3A. When labeled DS oligonucleotide probe containing the wild-type MTTP promoter $(-126$ to $-92 \mathrm{bp}$, wtMTTP) was incubated with HepG2 nuclear extract, two discrete DNA-protein complexes were detected (Fig. 3B, lane 1). Formation of both complexes was inhibited after the addition of a

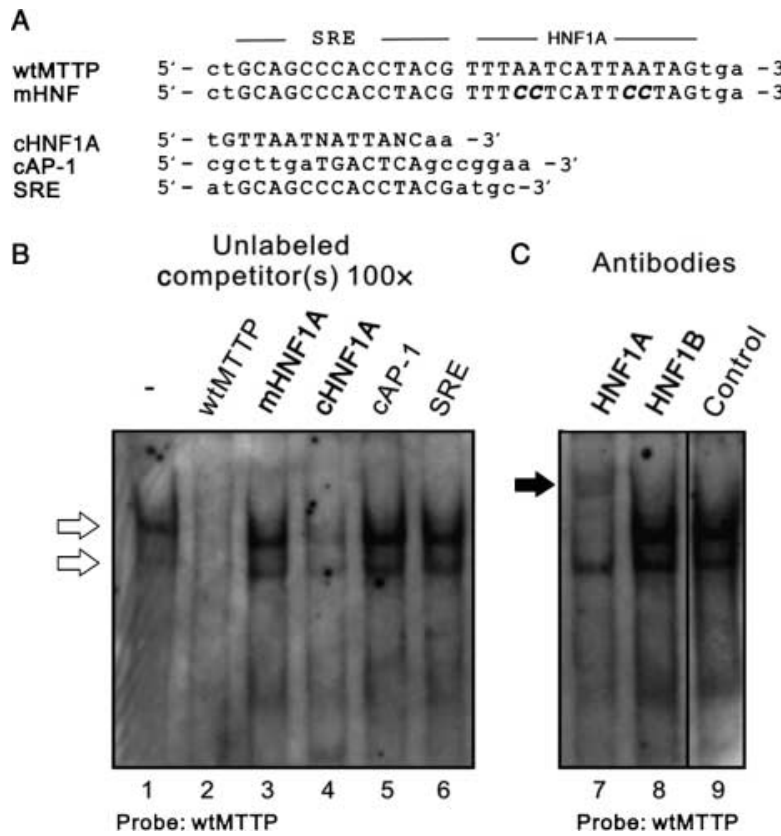

Figure 3 HNF1A binds to the HNF1A site in the MTTP promoter. (A) Oligonucleotides used in the EMSA. Lower case letters represent the flanking sequences, while upper case letters represent the sequence elements as indicated. The inactivating mutated region of the HNF1A site is shown in italics. (B) EMSA. The labeled probe of wild-type MTTP promoter sequence $(-126$ to $-92 \mathrm{bp}$ ) containing HNF1A site (wtMTTP) was incubated with $5 \mu \mathrm{g}$ nuclear extract of HepG2 cells for 30 min in the absence or presence of 100 -fold molar excess competitor as indicated. The specific protein-DNA complex was indicated by open arrow. wtMTTP, wild-type MTTP promoter; mHNF, MTTP promoter with mutated HNF1A; cHNF1A, consensus HNF1A element; CAP-1, consensus AP-1 element; SRE, SRE element. (C) Supershift EMSA. Nuclear extract from HepG2 cells was incubated with the indicated antibodies for $10 \mathrm{~min}$ at room temperature before addition of the labeled probe (wtMTTP). The supershift signal was indicated by solid arrow. 100-fold molar excess of unlabelled wtMTTP oligonucleotide competitor (Fig. 3B, lane 2). Remarkably, the same effect was observed with the addition of molar excess competitor containing consensus HNF1A element (cHNF1A; Fig. 3B, lane 4) but not with the competitor containing a mutated version of the HNF1A (mHNF1A; Fig. 3B, lane 3). Since there is an adjacent SRE element and a putative AP-1 site $(-109$ to $-103 \mathrm{bp})$ reside within this NIRE element $(-110$ to $-98 \mathrm{bp}$ ), we also tested the effect of oligonucleotide competitors containing the consensus AP-1 element (cAP-1; Fig. 3B, lane 5) and the SRE (Fig. 3B, lane 6). Results clearly showed that competition of these unlabeled oligonucleotides could not displace any labeled complexes, suggesting that neither the SREbinding protein nor AP-1 protein was directly associated with this NIRE element in the MTTP promoter.

To determine the identity of binding proteins, we pre-incubated the nuclear extract with antibodies against HNF1A, HNF1B, or UCP1 (negative control) prior to the addition of oligonucleotide probe. As shown in Fig. 3C, the supershifted/disrupted signal was exclusive to the HNF1A antibody treatment, indicating the presence of HNF1A in the complex and supporting the notion that this NIRE contains a functional HNF1A element, to which HNF1A binds.

Insulin signals the suppression of MTTP transcription through the MAPK ${ }^{\mathrm{MAPK} 1}$ pathway (Au et al. 2003). We therefore asked whether AP-1, the downstream effector transcription factor complex, is involved in insulin-mediated MTTP suppression. AP-1 does not bind directly to the MTTP promoter, as shown in EMSA assay in which the AP-1 site was used as probe (Fig. 3B, Lane 5). To test whether FOS and JUN, the two components of AP-1, are present on the NIRE when MTTP promoter is under the effect of insulin, we performed EMSA supershift experiments using FOS and JUN antibodies. FOS/JUN antibodies were preincubated with the nuclear extract from insulin-treated HepG2 cells, before the extract was incubated with label HNF1A element probe. Surprisingly, FOS antibody, but not JUN antibody, supershifted the DNA-protein complex (Fig. 4A, lanes 1 and 3). However, we cannot exclude the possibility that JUN is present in the complex since the epitopes recognized by the antibodies may be masked by the formation of the complex. The protein-protein interaction between HNF1A and FOS was confirmed by co-immunoprecipitation assay (Fig. 4B).

\section{Expression of HNF1A reconstitutes the basal activity and the insulin response of the MTTP promoter in HNF1A-deficient TM4SF1 myocytes}

Unlike HepG2 cells that express HNF1A (De et al. 1987) and MTTP (Wetterau et al. 1997), TM4SF1 muscle cells 


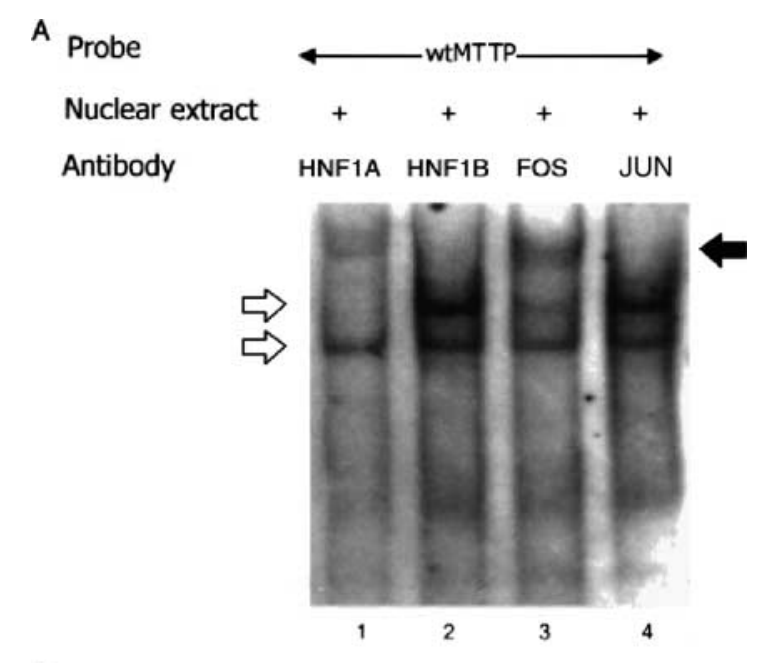

B

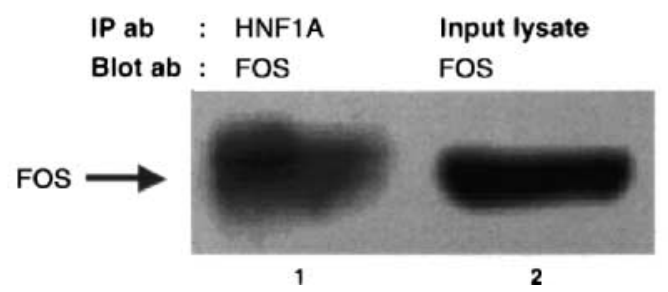

Figure 4 FOS is a component of the HNF1A complex formed on MTTP promoter upon insulin treatment. (A) EMSA supershift assay to investigate the component of the complex. About $5 \mu \mathrm{g}$ of nuclear extract was prepared from HepG2 cells treated with $100 \mathrm{nM}$ insulin for $24 \mathrm{~h}$ and was pre-incubated with $2 \mu \mathrm{g}$ indicated antibodies for $45 \mathrm{~min}$ prior to the addition of the probe. The mixture was resolved in $5 \%$ native gel and the probe was detected by chemiluminescence. White arrows, HNF1A complex; black arrow, supershifted complexes. (B) Co-immunoprecipitation of HNF1A and FOS. About $50 \mu \mathrm{g}$ of HepG2 nuclear extract was incubated with $2 \mu \mathrm{g}$ HNF1A antibody bound with agarose beads. The immunoprecipitated complex was resolved by $12 \%$ SDS-PAGE (lane 1) and immunoblotted against FOS antibody. The input lysate was loaded as reference (lane 2).

are deficient in expressing neither gene. Therefore, we transfected TM4SF1 cells with MTTP promoter/luciferase reporter construct (MTTP-250) in order to determine whether HNF1A is able to reconstitute the negative insulin responsiveness of the MTTP promoter. As shown in Fig. 5A, enforced expression of HNF1A in transfected TM4SF1 cells, confirmed by western blot, produced a dramatic fourfold increase in MTTP promoter activity, and this activity was significantly attenuated $(40 \%)$ by insulin treatment. By contrast, cells transfected with MTTP promoter with mutated HNF1A binding motif did not exhibit these effects. Also, forced expression of HNF1B could reconstitute neither MTTP promoter activity nor insulin responsiveness in TM4SF1 cells (Fig. 5B). This study fortified the critical role of HNF1A, but not HNF1B, in both basal activity and negative insulin response of the MTTP promoter.

\section{Consensus HNF1A element also displays negative insulin responsiveness}

Next, we asked whether the consensus HNF1A element displays negative insulin effect as its sequence (GTTAATNATTANC) differs slightly from that of the NIRE in MTTP promoter. We replaced this NIRE site with consensus (cHNF1A) or mutated HNF1A (mHNF1A) sequence in both native and fusion promoters, and tested their insulin responsiveness in HepG2 cells. In native MTTP promoter (Fig. 6A), replacement with cHNF1A element (MTTP-250cHNF1A) maintained the insulin responsiveness and basal activation activity, while mHNF1A element (MTTP-250-mHNF1A) abrogated both effects. In fusion promoters (Fig. 6B), both HNF1A (MTTP-reg and $1 \times$ HNF1A) and cHNF1A element (MTTP-reg-cHNF and $1 \times \mathrm{cHNF} 1 \mathrm{~A}$; with or without MTTP promoter $5^{\prime}$ flanking sequence respectively) exhibited the basal activation activity as well as negative insulin response, indicating that the consensus HNF1A element is functionally equivalent to NIRE.

\section{Discussion}

HNF1A binding element has been found in more than 100 liver-specific genes and has been established as a tissue-specific sequence element for gene transcription (Tronche et al. 1997, Odom et al. 2004). The binding protein, HNF1A, plays an important role in the activation of many liver-specific genes such as albumin through incorporation with co-activators in the formation of pre-initiation complexes (Schrem et al. 2002, Dohda et al. 2004). In this study, we provided the first evidence demonstrating that HNF1A element is more than a tissue-specific basal activity sequence. We showed that a functional HNF1A element ( -110 to $-98 \mathrm{bp}$ ) in the context of MTTP promoter is a novel NIRE. This conclusion is drawn based on the following observations. First, mutation of this element blunted the insulin responsiveness (Fig. 1B and C). Secondly, liverenriched factor HNF1A bound to this element (Fig. 3). Thirdly, the basal activity and the insulin responsiveness of MTTP promoter could be reconstituted in heterologous cells deficient in HNF1A when HNF1A was expressed (Fig. 5). Finally, minimal TEAD1 fusion promoter containing this element or consensus HNF1A element responded negatively toward insulin treatment in HepG2 cells (Figs 2 and 6).

We found that HNF1A element is necessary and sufficient to confer insulin responsiveness on MTTP promoter in contrast to an earlier study that suggested that the HNF1A element is an accessory factor in enhancing the inhibitory action of insulin on glucose-6-phosphatase $(G 6 P C)$ gene transcription 
A

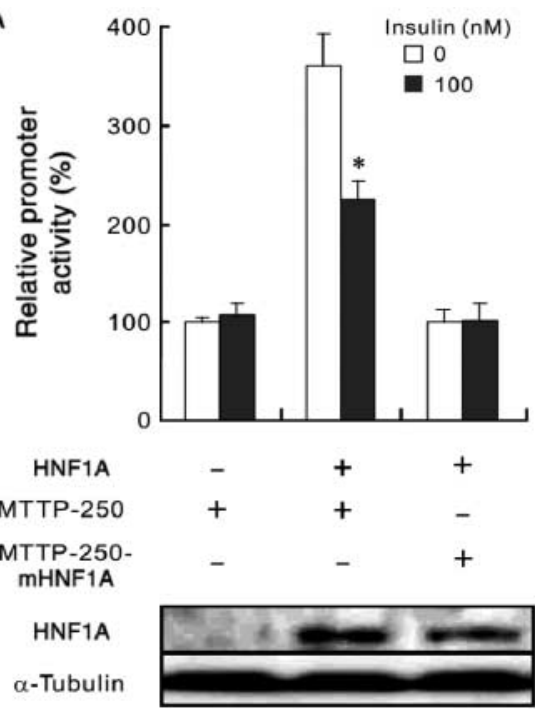

B
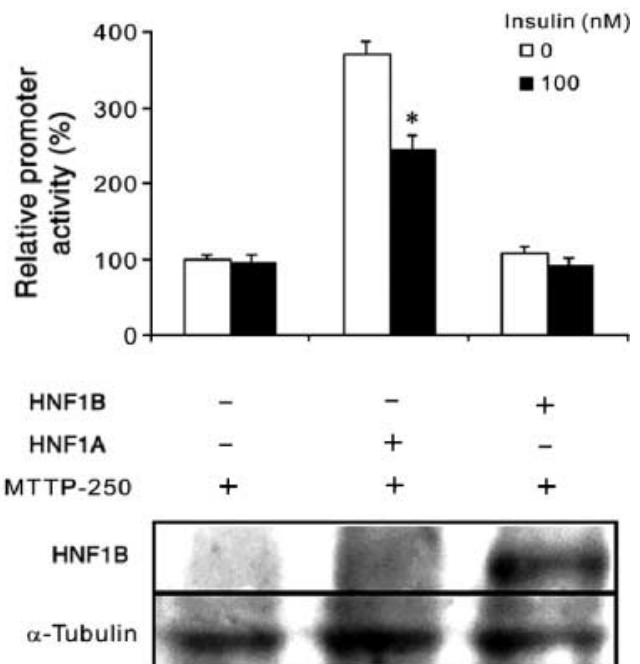

Figure 5 Expression of HNF1A, but not HNF1B, confers activation and negative insulin response on MTTP promoter in HNF1A-deficient TM4SF1 cells. (A and B) TM4SF1 muscle cells were co-transfected with the MTTP promoter-reporter construct, either wild type (MTTP-250) or HNF1A site inactivated (MTTP-250-mHNF1A), in the presence or absence of (A) HNF1A or (B) HNF1B expression vectors. After $16 \mathrm{~h}$ recovery in reduced serum medium, the cells were challenged with 0 or $100 \mathrm{nM}$ insulin in serum-free medium. Promoter activity obtained in the absence of HNF1A expression and insulin challenge was considered as reference (100\%). *Denotes significant difference from untreated counterpart $(P<0.05)$.

(Streeper et al. 1998). The discrepancy could be explained by the difference in the experimental design. The previous study fused HNF1A element with minimal TK promoter whose activity could be significantly stimulated by insulin. This may complicate the interpretation of the results since stimulation may mask the negative response mediated by HNF1A. In our design, we fused the HNF1A element with minimal TEAD1 promoter which displays better resistance toward insulin challenge. In addition, we normalized each transfection against an internal control using TEAD1 promoter to minimize the influence of nonspecific insulin effects. We clearly observed negative insulin response in the TEAD1 chimera with both single and tandem copies of the HNF1A element. We further confirmed the role of this element by reconstituting the negative insulin response of MTTP promoter in TM4SF1 myocytes expressing exogenous HNF1A. Taken together, these results fortified that HNF1A element alone can function as an NIRE.

The transcription of MTTP can be regulated by many agents. Besides insulin, conditions like sterol depletion (Sato et al. 1999) or exposure to endotoxins and cytokines (Navasa et al. 1998) may also lead to the suppression of MTTP gene transcription. Thus, injection of VWS or IL1A decreases MTTP mRNA level in Syrian hamster liver (Navasa et al. 1998). Interestingly, the IL1A-responsive element resides in -123 to -85 region, which also confers insulin responsiveness (Hagan et al. 1994), of the MTTP promoter. And a mutation study identified the HNF1A element as the only important IL1A-responsive element (Navasa et al. 1998). Hence, IL1A and insulin seem to share the same mechanism in down-regulating MTTP and this supports our finding that the HNF1A element is crucial for negative regulations of the MTTP gene.

The HNF1A element appears to possess two opposing functions, basal activation and repressive insulin response. Similar phenomenon has been observed in the NIRE found in gluconeogenic genes like phosphoenolpyruvate carboxykinase (PCK2; Hall et al. 2000, Yeagley et al. 2000, 2001, O'Brien et al. 2001) and G6PC (Streeper et al. 1998, Ayala et al. 1999, O'Brien et al. 2001). The negative insulin response exerted by the HNF1A element could be due to the recruitment of negative factor to or withdrawal of positive factor from the HNF1A complex as in the NIRE of PCK2 (Hall et al. 2000, Yeagley et al. 2000, 2001). In terms of upstream signaling, we previously demonstrated that the negative insulin effect on MTTP promoter is mediated by the MAPK $^{\text {erk }}$ signaling but not the phosphoinositide 3-kinases (PIK3C2A/B) pathway (Au et al. 2003). Therefore, the HNF1A-mediated repressive insulin response is distinct from that of the NIRE of PCK2, not only in the nuclear action but also in the cytosolic signaling. 
A

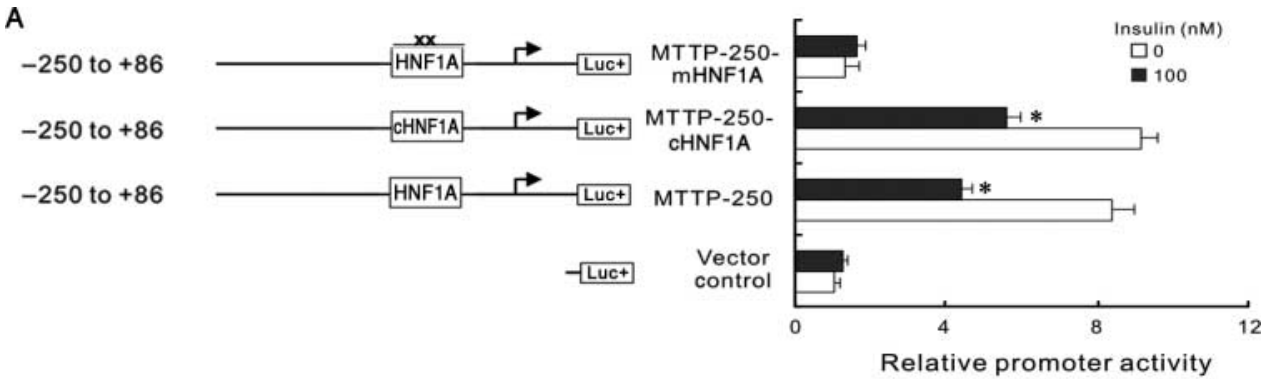

B
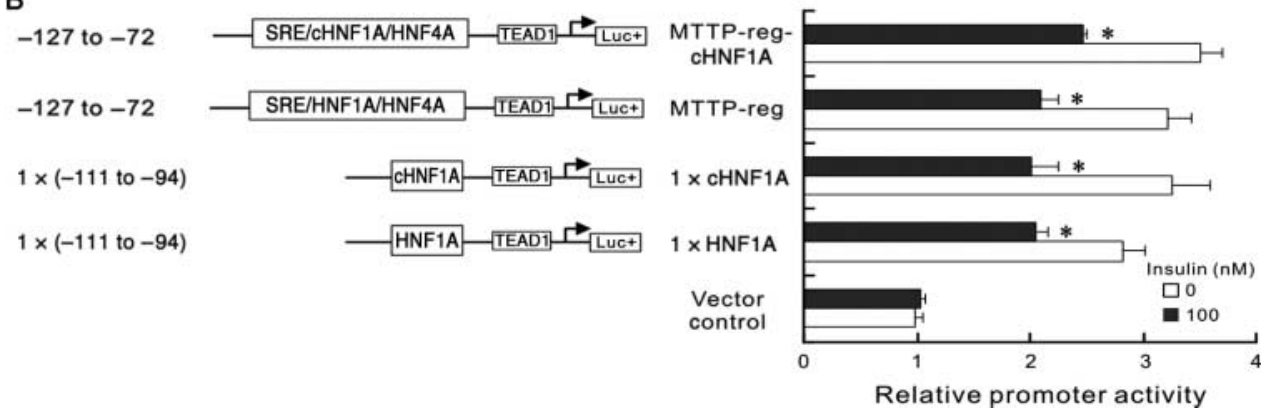

Figure 6 Consensus HNF1A element also displays basal activation activity and negative insulin response. (A) Native MTTP promoter cloned into SDHC-Basic vector. The sequence of HNF1A element in the MTTP promoter-reporter construct (MTTP-250, -250 to $+86 \mathrm{bp}$ ) was replaced with either consensus HNF1A sequence (MTTP-250-cHNF1A) or mutated sequences (MTTP-250-mHNF1A). (B) Fusion promoters using SDHC-promoter vector (contains minimal TEAD1 promoter). The HNF1A element in MTTP promoter sequence (MTTP-reg, -127 to $-72 \mathrm{bp}$ ) was replaced with consensus element (MTTP-reg-cHNF). Consensus HNF1A element or MTTP HNF1A element was fused with minimal TEAD1 promoter $(1 \times$ CHNF1A and $1 \times$ HNF1A respectively). HepG2 cells were transfected with these constructs, allowed to recover in reduced serum medium, and treated with or without $100 \mathrm{nM}$ insulin. Relative promoter activity was calculated as the ratio of normalized RPUSD1 of different treated or untreated promoters to that of the untreated vector control or empty vector. *Denotes significant difference from untreated counterpart $(P<0.05)$.

We have attempted to study the makeup of the HNF1A complex in order to understand the molecular mechanism of the transcription inhibitory effects of insulin on the MTTP gene. We showed that FOS interacts with HNF1A but more than that binds directly to DNA (Fig. 4). These data confirmed the involvement of MAPK signaling pathway. Moreover, it is possible that phosphorylation of the protein complex and subsequent nuclear exclusion of the phosphorylated complex are involved in mediating the transcription inhibitory effect of insulin. These phenomena have been recently reported in the insulin-dependent regulation of MTTP expression mediated by a forkhead transcription factor, forkhead box O1 (FOXO1; Kamagate $e$ al. 2008). However, the insulin signal transduction pathway of FOXO1 phosphorylation is PIK3C2A/B (Kamagate et al. 2008), instead of MAPK ${ }^{\text {erk }}$ signaling. Whether there is any coordination between the two signaling pathways is worth investigating.

Although HNF1A element on the MTTP promoter differs slightly from the consensus sequence, an exchange for consensus HNF1A sequence in the MTTP promoters did not alter the basal activation activity and insulin responsiveness. The same effect was observed in heterologous fusion promoter containing consensus HNF1A sequence and minimal TEAD1 promoter, indicating that these HNF1A elements are functionally equivalent (Fig. 6). This implies that the HNF1A-mediated negative insulin response is not specific to the MTTP gene but to genes containing HNF1A binding element. Since a panel of liver-specific genes implicated in diabetes contains HNF1A binding element, their potential regulations by insulin may offer important clues for the manifestation of diabetic metabolic syndromes.

We also showed in the present study that enforced expression of HNF1A, but not HNF1B, could restore the MTTP promoter activity and the negative insulin response in the HNF1A-deficient TM4SF1 cells (Fig. 5). Previous studies also demonstrated that HNF1A antagonizes (Ktistaki \& Talianidis 1997) or synergizes (Hu \& Perlmutter 1999, Sheena et al. 2005) gene transcription. Therefore, the functional role of HNF1A cannot be entirely replaced by HNF1B. As HNF1A plays an important role in the regulation of lipid homeostasis (Akiyama et al. 2000) and a deficiency of it causes 
maturity-onset diabetes of the young type 3 (HNF1A; Yamagata et al. 1996), we propose that the hepatic insulin signaling axis via HNF1A element may play a crucial role in the pathophysiology of diabetic dyslipidemia and HNF1A. Knowledge regarding the molecular mechanisms and the key factors involved in this axis may provide important insights into and new therapeutic targets for the treatment of diabetic metabolic syndromes.

\section{Declaration of interest}

The authors declare that there is no conflict of interest.

\section{Funding}

This work was supported by grants from the Research Grants Council of the Hong Kong Special Administrative Region, China (HKU7642/05M to M C L), and in part by Li Ka Shing Institute of Health Sciences (to H F K), Shanghai Metropolitan Fund for Research and Development (04JC14096), and Foundation of Guangzhou Science and Technology Bureau (2005Z1-E0131).

\section{References}

Akiyama TE, Ward JM \& Gonzalez FJ 2000 Regulation of the liver fatty acid-binding protein gene by hepatocyte nuclear factor lalpha (HNFlalpha). Alterations is fatty acid homeostasis in HNF-lalphadificient mice. Journal of Biological Chemistry 275 27117-27122.

Au WS, Kung HF \& Lin MC 2003 Regulation of microsomal triglyceride transfer protein gene by insulin in HepG2 cells: roles of MAPKerk and MAPKp38. Diabetes 52 1073-1080.

Austin MA, Talmud PJ, Luong LA, Haddad L, Day IN, Newman B, Edwards KL, Krauss RM \& Humphries SE 1998 Candidate-gene studies of the atherogenic lipoprotein phenotype: a sib-pair linkage analysis of $\mathrm{DZ}$ women twins. American Journal Human Genetics 62 406-419.

Ayala JE, Streeper RS, Desgrosellier JS, Durham SK, Suwanichkul A, Svitek CA, Goldman JK, Barr FG, Powell DR \& O’Brien RM 1999 Conservation of an insulin response unit between mouse and human glucose-6-phosphatase catalytic subunit gene promoters: transcription factor FKHR binds the insulin response sequence. Diabetes 48 1885-1889.

Bonham K, Ritchie SA, Dehm SM, Snyder K \& Boyd FM 2000 An alternative, human SRC promoter and its regulation by hepatic nuclear factor-1alpha. Journal of Biological Chemistry 275 37604-37611.

De SV, Ciliberto G, Hardon E, Paonessa G, Palla F, Lundberg L \& Cortese R 1987 Cis- and trans-acting elements responsible for the cell-specific expression of the human alpha 1-antitrypsin gene. EMBO Journal 6 2759-2766.

Dohda T, Kaneoka H, Inayoshi Y, Kamihira M, Miyake K \& Iijima S 2004 Transcriptional coactivators CBP and p300 cooperatively enhance HNF-1alpha-mediated expression of the albumin gene in hepatocytes. Journal of Biochemistry 136 313-319.

Hagan DL, Kienzle B, Jamil H \& Hariharan N 1994 Transcriptional regulation of human and hamster microsomal triglyceride transfer protein genes. Cell type-specific expression and response to metabolic regulators. Journal of Biological Chemistry 269 28737-28744.

Hall RK, Yamasaki T, Kucera T, Waltner-Law M, O'Brien R \& Granner DK 2000 Regulation of phosphoenolpyruvate carboxykinase and insulin-like growth factor-binding protein-1 gene expression by insulin. The role of winged helix/forkhead proteins. Journal of Biological Chemistry 275 30169-30175.

Hu C \& Perlmutter DH 1999 Regulation of alphal-antitrypsin gene expression in human intestinal epithelial cell line caco-2 by HNFlalpha and HNF-4. American Journal of Physiology 276 G1181-G1194.

Hussain MM, Iqbal J, Anwar K, Rava P \& Dai K 2003 Microsomal triglyceride transfer protein: a multifunctional protein. Frontiers in Bioscience 8 s500-s506.

Kamagate A, Qu S, Perdomo G, Su D, Kim DH, Slusher S, Meseck M \& Dong HH 2008 FoxO1 mediates insulin-dependent regulation of hepatic VLDL production in mice. Journal of Clinical Investigation 118 2347-2364.

Ktistaki E \& Talianidis I 1997 Modulation of hepatic gene expression by hepatocyte nuclear factor 1. Science 277 109-112.

Kuriyama H, Yamashita S, Shimomura I, Funahashi T, Ishigami M, Aragane K, Miyaoka K, Nakamura T, Takemura K, Man Z et al. 1998 Enhanced expression of hepatic acyl-coenzyme A synthetase and microsomal triglyceride transfer protein messenger RNAs in the obese and hypertriglyceridemic rat with visceral fat accumulation. Hepatology 27 557-562.

Ledmyr H, Karpe F, Lundahl B, McKinnon M, Skoglund-Andersson C \& Ehrenborg E 2002 Variants of the microsomal triglyceride transfer protein gene are associated with plasma cholesterol levels and body mass index. Journal of Lipid Research 43 51-58.

Ledmyr H, McMahon AD, Ehrenborg E, Nielsen LB, Neville M, Lithell H, MacFarlane PW, Packard CJ \& Karpe F 2004 The microsomal triglyceride transfer protein gene-493T variant lowers cholesterol but increases the risk of coronary heart disease. Circulation 109 2279-2284

Ledmyr H, Ottosson L, Sunnerhagen M \& Ehrenborg E 2006 The Ile128Thr polymorphism influences stability and ligand binding properties of the microsomal triglyceride transfer protein. Journal of Lipid Research 47 1378-1385.

Lin MC, Arbeeny C, Bergquist K, Kienzle B, Gordon DA \& Wetterau JR 1994 Cloning and regulation of hamster microsomal triglyceride transfer protein. The regulation is independent from that of other hepatic and intestinal proteins which participate in the transport of fatty acids and triglycerides. Journal of Biological Chemistry $26929138-29145$.

Lin MC, Gordon D \& Wetterau JR 1995 Microsomal triglyceride transfer protein (MTP) regulation in HepG2 cells: insulin negatively regulates MTP gene expression. Journal of Lipid Research 36 1073-1081.

Lin MC, Li JJ, Wang EJ, Princler GL, Kauffman FC \& Kung HF 1997 Ethanol down-regulates the transcription of microsomal triglyceride transfer protein gene. FASEB Journal 11 1145-1152.

Mounier C \& Posner BI 2006 Transcriptional regulation by insulin: from the receptor to the gene. Canadian Journal of Physiology and Pharmacology 84 713-724.

Navasa M, Gordon DA, Hariharan N, Jamil H, Shigenaga JK, Moser A, Fiers W, Pollock A, Grunfeld C \& Feingold KR 1998 Regulation of microsomal triglyceride transfer protein mRNA expression by endotoxin and cytokines. Journal of Lipid Research 39 1220-1230.

O'Brien RM, Streeper RS, Ayala JE, Stadelmaier BT \& Hornbuckle LA 2001 Insulin-regulated gene expression. Biochemical Society Transactions 29 552-558.

Odom DT, Zizlsperger N, Gordon DB, Bell GW, Rinaldi NJ, Murray HL, Volkert TL, Schreiber J, Rolfe PA, Gifford DK et al. 2004 Control of pancreas and liver gene expression by HNF transcription factors. Science 303 1378-1381.

Ono M, Hosoe Y, Azuma S, Shoji M, Nara K, Kondo N, Shiba T \& Takamatsu N 2001 HNF-1 regulates the liver-specific transcription of the chipmunk HP-20 gene. Gene 277 121-127.

Rainwater DL, Almasy L, Blangero J, Cole SA, VandeBerg JL, MacCluer JW \& Hixson JE 1999 A genome search identifies major quantitative 
trait loci on human chromosomes 3 and 4 that influence cholesterol concentrations in small LDL particles. Arteriosclerosis, Thrombosis, and Vascular Biology 19 777-783.

Rubin D, Schneider-Muntau A, Klapper M, Nitz I, Helwig U, Folsch UR, Schrezenmeir J \& Doring F 2008 Functional analysis of promoter variants in the microsomal triglyceride transfer protein (MTTP) gene. Human Mutation 29 123-129.

Ryffel GU 2001 Mutations in the human genes encoding the transcription factors of the hepatocyte nuclear factor (HNF) 1 and HNF4 families: functional and pathological consequences. Journal of Molecular Endocrinology 27 11-29.

Saltiel AR \& Kahn CR 2001 Insulin signalling and the regulation of glucose and lipid metabolism. Nature 414 799-806.

Sato R, Miyamoto W, Inoue J, Terada T, Imanaka T \& Maeda M 1999 Sterol regulatory element-binding protein negatively regulates microsomal triglyceride transfer protein gene transcription. Journal of Biological Chemistry 274 24714-24720.

Schrem H, Klempnauer J \& Borlak J 2002 Liver-enriched transcription factors in liver function and development. Part I: the hepatocyte nuclear factor network and liver-specific gene expression. Pharmacological Reviews 54 129-158.

Sharp D, Ricci B, Kienzle B, Lin MC \& Wetterau JR 1994 Human microsomal triglyceride transfer protein large subunit gene structure. Biochemistry 33 9057-9061.

Sheena V, Hertz R, Nousbeck J, Berman I, Magenheim J \& Bar-Tana J 2005 Transcriptional regulation of human microsomal triglyceride transfer protein by hepatocyte nuclear factor-4alpha. Journal of Lipid Research 46 328-341.

Shoulders CC \& Shelness GS 2005 Current biology of MTP: implications for selective inhibition. Current Topics in Medicinal Chemistry 5 283-300.

Streeper RS, Eaton EM, Ebert DH, Chapman SC, Svitek CA \& O’Brien RM 1998 Hepatocyte nuclear factor-1 acts as an accessory factor to enhance the inhibitory action of insulin on mouse glucose-6phosphatase gene transcription. PNAS 95 9208-9213.
Taghibiglou C, Carpentier A, Van Iderstine SC, Chen B, Rudy D, Aiton A, Lewis GF \& Adeli K 2000 Mechanisms of hepatic very low density lipoprotein overproduction in insulin resistance. Evidence for enhanced lipoprotein assembly, reduced intracellular ApoB degradation, and increased microsomal triglyceride transfer protein in a fructose-fed hamster model. Journal of Biological Chemistry 275 8416-8425.

Tronche F, Ringeisen F, Blumenfeld M, Yaniv M \& Pontoglio M 1997 Analysis of the distribution of binding sites for a tissue-specific transcription factor in the vertebrate genome. Journal of Molecular Biology 266 231-245.

Wetterau JR, Lin MC \& Jamil H 1997 Microsomal triglyceride transfer protein. Biochimica et Biophysica Acta 1345 136-150.

Yamagata K, Oda N, Kaisaki PJ, Menzel S, Furuta H, Vaxillaire M, Southam L, Cox RD, Lathrop GM, Boriraj VV et al. 1996 Mutations in the hepatocyte nuclear factor-1alpha gene in maturity-onset diabetes of the young (MODY3). Nature 384 455-458.

Yeagley D, Moll J, Vinson CA \& Quinn PG 2000 Characterization of elements mediating regulation of phosphoenolpyruvate carboxykinase gene transcription by protein kinase $\mathrm{A}$ and insulin. Identification of a distinct complex formed in cells that mediate insulin inhibition. Journal of Biological Chemistry 275 17814-17820.

Yeagley D, Guo S, Unterman T \& Quinn PG 2001 Gene- and activationspecific mechanisms for insulin inhibition of basal and glucocorticoid-induced insulin-like growth factor binding protein-1 and phosphoenolpyruvate carboxykinase transcription. Roles of forkhead and insulin response sequences. Journal of Biological Chemistry 276 33705-33710.

Received in final form 15 July 2008

Accepted 8 August 2008

Made available online as an Accepted Preprint 12 August 2008 